\title{
Monomial Ideals and Planar Graphs
}

\author{
Ezra Miller and Bernd Sturmfels \\ Department of Mathematics, University of California, Berkeley CA 94720, USA \\ enmiller@math.berkeley.edu, bernd@math.berkeley.edu
}

\begin{abstract}
Gröbner basis theory reduces questions about systems of polynomial equations to the combinatorial study of monomial ideals, or staircases. This article gives an elementary introduction to current research in this area. After reviewing the bivariate case, a new correspondence is established between planar graphs and minimal resolutions of monomial ideals in three variables. A brief guide is given to the literature on complexity issues and monomial ideals in four or more variables.
\end{abstract}

\section{Introduction}

A monomial ideal $M$ is an ideal generated by monomials $x_{1}^{i_{1}} x_{2}^{i_{2}} \cdots x_{n}^{i_{n}}$ in a polynomial ring $\mathbb{K}\left[x_{1}, x_{2}, \ldots, x_{n}\right]$. Monomial ideals are ubiquitous in the study of Gröbner bases. For instance, if $I=\left\langle x^{4}+y^{4}-1, x^{7}+y^{7}-2\right\rangle$ then its initial ideal with respect to the total degree term order equals $M=\left\langle x^{4}, x^{3} y^{4}, x y^{7}, y^{10}\right\rangle$. The ideal $I$ has 28 distinct complex roots, corresponding to the 28 monomials $x^{i} y^{j}$ not in $M$, that is, to the 28 lattice points under the staircase depicting $M$ :

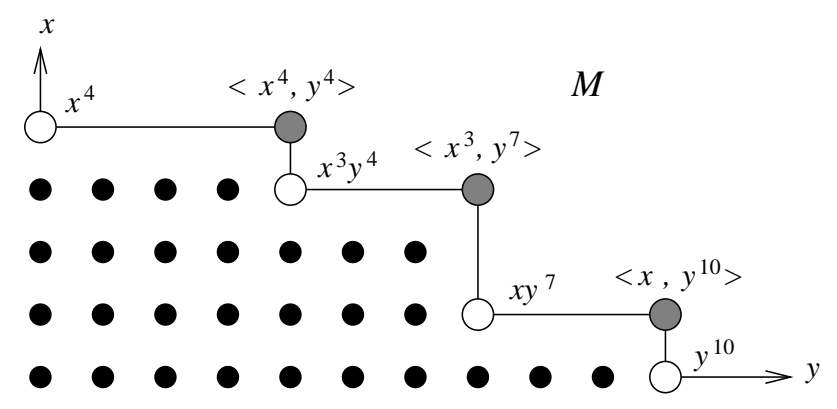

Fig. 1. The monomial ideal $M=\left\langle x^{4}, x^{3} y^{4}, x y^{7}, y^{10}\right\rangle$, with its generators (white circles), standard monomials (black dots), and irreducible components (shaded circles)

At any stage in Buchberger's algorithm for computing Gröbner bases, one considers the S-pairs among the current polynomials and removes those which are redundant [7]. The minimal $S$-pairs define a graph $G_{M}$ on the generators of any monomial ideal $M$. Our aim is to study this graph, and to give an elementary introduction to recent work on computing the following objects associated to $M$ :

i. the Hilbert series of $M$, i.e. the formal sum of all monomials not in $M$; 
ii. the minimal resolution of $M$ by free modules over the polynomial ring;

iii. the primary decomposition and the irreducible decomposition of $M$.

For a first introduction to these problems see $[10, \S 9.2]$ and [11, Exercises 3.8 and 17.11]. Their importance for Buchberger's Algorithm was emphasized by Möller and Mora in [16]. Research papers describing effective algorithms are [4] and [6] for problem (i), [9] and [14] for problem (ii), and [8] for problem (iii).

Our point of view differs from these sources. We regard (i), (ii), (iii) as the same problem and we develop combinatorial tools for presenting structured solutions. It is our belief that this approach will ultimately lead to faster algorithms. This paper is organized as follows. The easy solution for $n=2$ variables will be reviewed in Section 2. In Sections 3 and 4 we present the generalization to the case of three variables. We shall see that the S-pair graphs $G_{M}$ for $n=3$ are precisely the planar graphs. Section 5 summarizes what is known for $n \geq 4$.

\section{Two Variables}

Let us begin by answering the three computational questions for our example.

1. The Hilbert series of the monomial ideal $M=\left\langle x^{4}, x^{3} y^{4}, x y^{7}, y^{10}\right\rangle$ equals

$$
\frac{1-x^{4}-x^{3} y^{4}-x y^{7}-y^{10}+x^{4} y^{4}+x^{3} y^{7}+x y^{10}}{(1-x)(1-y)} .
$$

This expression equals the sum of the 28 standard monomials in Figure 1.

2. The minimal free resolution of $M$ is the following exact sequence of modules,

$$
0 \longrightarrow \mathbb{K}[x, y]^{3} \stackrel{\partial_{1}}{\longrightarrow} \mathbb{K}[x, y]^{4} \stackrel{\partial_{0}}{\longrightarrow} M \longrightarrow 0,
$$

where, in matrix notation, $\partial_{1}=\left(\begin{array}{ccc}y^{4} & 0 & 0 \\ -x & y^{3} & 0 \\ 0 & -x^{2} & y^{3} \\ 0 & 0 & -x\end{array}\right)$ and $\partial_{0}=\left(\begin{array}{llll}x^{4} & x^{3} y^{4} & x y^{7} & y^{10}\end{array}\right)$.

3. The ideal $M$ is $\langle x, y\rangle$-primary and its irreducible decomposition equals

$$
M=\left\langle x^{4}, y^{4}\right\rangle \cap\left\langle x^{3}, y^{7}\right\rangle \cap\left\langle x, y^{10}\right\rangle . \quad \text { (see Figure 1) }
$$

It is not difficult to extend this to an arbitrary monomial ideal in two variables,

$$
M=\left\langle x^{a_{1}} y^{b_{1}}, x^{a_{2}} y^{b_{2}}, \ldots, x^{a_{r}} y^{b_{r}}\right\rangle \text {, where } a_{1}>\cdots>a_{r} \text { and } b_{1}<\cdots<b_{r} .
$$

Proposition 1. The following holds for any monomial ideal $M$ in two variables:

1. The Hilbert series of $M$ equals

$$
\sum\left\{x^{i} y^{j}: x^{i} y^{j} \notin M\right\}=\frac{1-\sum_{j=1}^{r} x^{a_{j}} y^{b_{j}}+\sum_{i=1}^{r-1} x^{a_{i}} y^{b_{i+1}}}{(1-x)(1-y)} .
$$


2. The minimal free resolution of $M$ equals

$$
0 \longrightarrow \mathbb{K}[x, y]^{r-1} \stackrel{\partial_{1}}{\longrightarrow} \mathbb{K}[x, y]^{r} \stackrel{\partial_{0}}{\longrightarrow} M \longrightarrow 0,
$$

where $\partial_{0}$ is the canonical map and $\partial_{1}$ is given on standard basis vectors by $\partial_{1}\left(e_{i}\right)=y^{b_{i+1}-b_{i}} \cdot e_{i}-x^{a_{i}-a_{i+1}} \cdot e_{i+1}$.

3. The irreducible decomposition of $M$ equals

$$
M=\left\langle y^{b_{1}}\right\rangle \cap\left\langle x^{a_{1}}, y^{b_{2}}\right\rangle \cap\left\langle x^{a_{2}}, y^{b_{3}}\right\rangle \cap \cdots \cap\left\langle x^{a_{r-1}}, y^{b_{r}}\right\rangle \cap\left\langle x^{a_{r}}\right\rangle,
$$

where the first or last component are to be removed if $b_{1}=0$ or $a_{r}=0$.

This implies that for monomials in two variables only consecutive $S$-pairs matter.

Corollary 1. When applying Buchberger's Algorithm to $r$ polynomials in two variables, it suffices to form the $r-1$ consecutive $S$-pairs, instead of all $\left(\begin{array}{l}r \\ 2\end{array}\right)$ pairs.

\section{Three Variables: the Generic Case}

This section gives a combinatorial introduction to the results in the article [3]. The Hilbert series of any monomial ideal $M=\left\langle m_{1}, m_{2}, \ldots, m_{r}\right\rangle$ in the polynomial ring $\mathbb{K}\left[x_{1}, \ldots, x_{n}\right]$ can be written using Inclusion-Exclusion as follows. For $I \subseteq\{1,2, \ldots, r\}$ let $m_{I}$ denote the least common multiple of $\left\{m_{i}: i \in I\right\}$.

Proposition 2. The Hilbert series of $M$ equals

$$
\sum\left\{x_{1}^{i_{1}} x_{2}^{i_{2}} \cdots x_{n}^{i_{n}} \notin M\right\}=\frac{1}{\prod_{i=1}^{n}\left(1-x_{i}\right)} \cdot \sum_{I \subseteq\{1,2, \ldots, r\}}(-1)^{|I|} \cdot m_{I} .
$$

Unfortunately this formula is useless for our problem (i), because the number of summands is an exponential function in $r$. But almost all summands cancel.

In this section we show that for $n=3$ the true number of summands in the numerator of the Hilbert series of $M$ is a linear function in $r$. We write $x, y, z$ for the variables. To simplify our discussion, we first assume that $r \geq 4$ and $M$ is generic in the following sense: if $m_{i}=x^{a_{i}} y^{b_{i}} z^{c_{i}}$ and $m_{j}=x^{a_{j}} y^{b_{j}} z^{c_{j}}$ are minimal generators of $M$ neither of which is a power of a single variable, then $a_{i} \neq a_{j}, b_{i} \neq b_{j}, c_{i} \neq c_{j}$. In Section 4 we shall remove this hypothesis.

We define a graph $G_{M}$ with vertices $1,2, \ldots, r$ by declaring $\{i<j\}$ an edge whenever $m_{i j}=\operatorname{lcm}\left(m_{i}, m_{j}\right)$ is not a multiple of $m_{k}$ for any $k \in\{1, \ldots, r\} \backslash\{i, j\}$. Let $T_{M}$ be the set of triples $\{i<j<k\}$ which form a triangle $\{i, j\},\{i, k\},\{j, k\}$ in $G_{M}$, and which do not consist entirely of powers $x^{a_{i}}, y^{b_{j}}, z^{c_{k}}$ of the variables. Consider the free module $\mathbb{K}[x, y, z]^{T_{M}}$ with basis $\left\{e_{i j k}:\{i, j, k\} \in T_{M}\right\}$, the free module $\mathbb{K}[x, y, z]^{G_{M}}$ with basis $\left\{e_{i j}:\{i, j\} \in G_{M}\right\}$, and the linear maps

$$
\begin{aligned}
& \partial_{1}: \mathbb{K}[x, y, z]^{G_{M}} \rightarrow \mathbb{K}[x, y, z]^{r}, e_{i j} \mapsto \frac{m_{i j}}{m_{j}} \cdot e_{j}-\frac{m_{i j}}{m_{i}} \cdot e_{i}, \\
& \partial_{2}: \mathbb{K}[x, y, z]^{T_{M}} \rightarrow \mathbb{K}[x, y, z]^{G_{M}}, e_{i j k} \mapsto \frac{m_{i j k}}{m_{j k}} \cdot e_{j k}-\frac{m_{i j k}}{m_{i k}} \cdot e_{i k}+\frac{m_{i j k}}{m_{i j}} \cdot e_{i j} .
\end{aligned}
$$


Theorem 1. Let $M$ be a generic monomial ideal in $\mathbb{K}[x, y, z]$.

1. The Hilbert series of $M$ equals

$$
\frac{1-\sum_{i=1}^{r} m_{i}+\sum_{\{i, j\} \in G_{M}} m_{i j}-\sum_{\{i, j, k\} \in T_{M}} m_{i j k}}{(1-x)(1-y)(1-z)} .
$$

2. The minimal free resolution of $M$ equals

$$
0 \longrightarrow \mathbb{K}[x, y, z]^{T_{M}} \stackrel{\partial_{2}}{\longrightarrow} \mathbb{K}[x, y, z]^{G_{M}} \stackrel{\partial_{1}}{\longrightarrow} \mathbb{K}[x, y, z]^{r} \stackrel{\partial_{0}}{\longrightarrow} M \longrightarrow 0 .
$$

3. If $M$ is artinian then the irreducible decomposition equals

$$
M=\bigcap_{\{i, j, k\} \in T_{M}}\left\langle x^{\operatorname{deg}_{x}\left(m_{i j k}\right)}, y^{\operatorname{deg}_{y}\left(m_{i j k}\right)}, z^{\operatorname{deg}_{z}\left(m_{i j k}\right)}\right\rangle .
$$

Here $M$ is called artinian if some power of each variable is in $M$, or equivalently, if the number of standard monomials is finite. The non-artinian case can be reduced to the artinian case by considering $M+\left\langle x^{m}, y^{m}, z^{m}\right\rangle$ for $m \gg 0$.

We illustrate Theorem 4 for the generic artinian monomial ideal

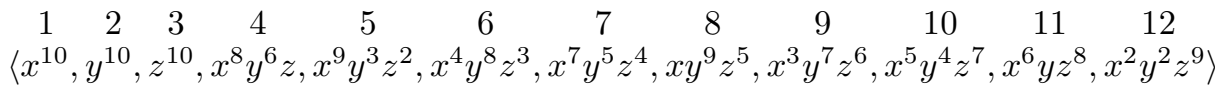

$$
\begin{aligned}
& =\left\langle x^{10}, y^{10}, z\right\rangle \cap\left\langle x^{8}, y^{10}, z^{3}\right\rangle \cap\left\langle x^{7}, y^{8}, z^{6}\right\rangle \cap\left\langle x^{9}, y^{5}, z^{7}\right\rangle \cap\left\langle x^{7}, y^{7}, z^{7}\right\rangle \cap \\
& \left\langle x^{10}, y^{3}, z^{8}\right\rangle \cap\left\langle x^{9}, y^{4}, z^{8}\right\rangle \cap\left\langle x^{5}, y^{7}, z^{9}\right\rangle \cap\left\langle x^{3}, y^{9}, z^{9}\right\rangle \cap\left\langle x^{10}, y, z^{10}\right\rangle \cap \\
& \left\langle x^{2}, y^{9}, z^{10}\right\rangle \cap\left\langle x, y^{10}, z^{10}\right\rangle \cap\left\langle x^{8}, y^{8}, z^{4}\right\rangle \cap\left\langle x^{9}, y^{6}, z^{4}\right\rangle \cap\left\langle x^{4}, y^{10}, z^{5}\right\rangle \cap \\
& \left\langle x^{4}, y^{9}, z^{6}\right\rangle \cap\left\langle x^{6}, y^{4}, z^{9}\right\rangle \cap\left\langle x^{10}, y^{6}, z^{2}\right\rangle \cap\left\langle x^{6}, y^{2}, z^{10}\right\rangle
\end{aligned}
$$
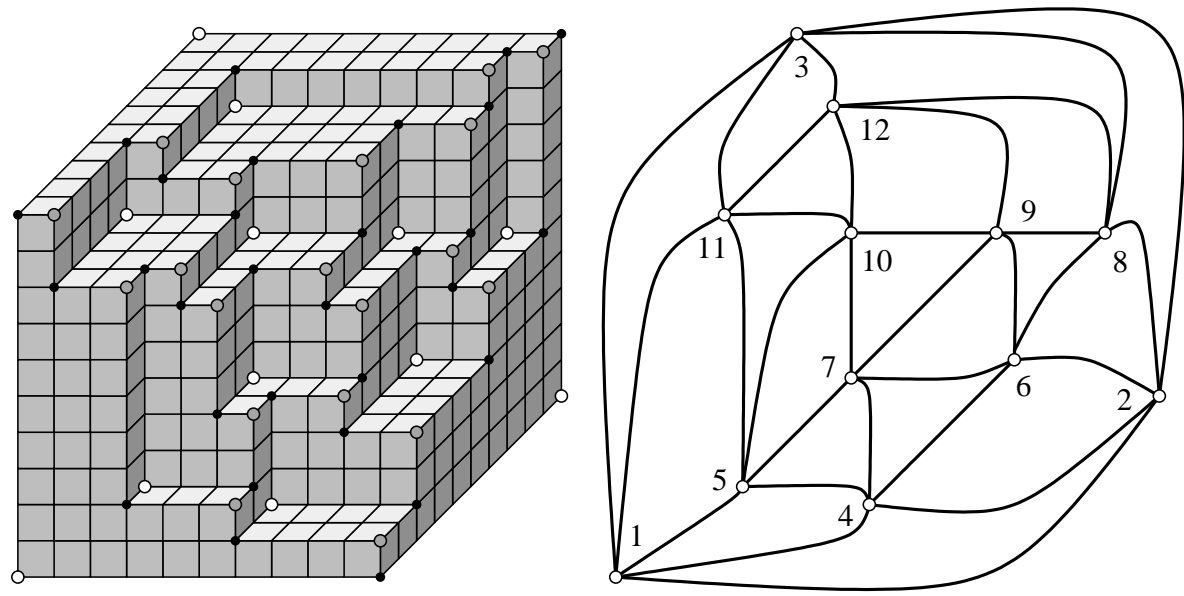

Fig. 2. A generic monomial ideal with 12 generators (white circles), 30 minimal S-pairs (black dots/edges), and 19 irreducible components (shaded circles/triangular regions) 
The right picture shows the graph $G_{M}$. Its triangles correspond to irreducible components; for instance, the last component $\left\langle x^{6}, y^{2}, z^{10}\right\rangle$ arises from the triangle $\{3,11,12\} \in T_{M}$. The numerator of the Hilbert series has $62=1+12+30+19$ summands, one for each vertex, edge and region of the planar graph $G_{M}$.

We are now ready to establish the connection promised in this paper's title.

Theorem 2. The S-pair graph $G_{M}$ of a generic monomial ideal $M$ in three variables is planar. If $M$ is artinian then $\left(G_{M}, T_{M}\right)$ is triangulation of a triangle.

Proof sketch. It suffices to consider artinian ideals $M$, since one can throw in high powers $x^{m}, y^{m}, z^{m}$, compute the S-pair graph, and then obtain $G_{M}$ by deleting any edges involving $x^{m}, y^{m}$, or $z^{m}$. We may further assume that each other generator of $M$ is divisible by $x y z$. The idea is to construct a 3-dimensional polytope whose edge graph equals $G_{M}$. Our claim follows because edge graphs of 3 -polytopes are planar. For instance, Figure 2 is the edge graph of an icosahedron.

Fix a large real number $t \gg 0$. Let $P_{t}$ denote the convex hull of the points $\left(t^{a_{i}}, t^{b_{i}}, t^{c_{i}}\right) \in \mathbb{R}^{3}$ as $\left(a_{i}, b_{i}, c_{i}\right)$ runs over the exponents on the minimal generators $x^{a_{i}} y^{b_{i}} z^{c_{i}}$ of $M$. Each such point is a vertex of $P_{t}$, and the edge graph of $P_{t}$ is independent of $t$. We call $P_{t}$ the hull polytope of $M$. One shows that every edge $E$ of $G_{M}$ is an edge of the hull polytope by producing a linear functional which is minimized on $P_{t}$ along $E$ (this does not use genericity of $M$ ). Finally, the genericity of $M$ is used to show that every edge of $P_{t}$ is obtained in this fashion.

Theorem 2 gives an answer to our questions (i), (ii), (iii) even if $M$ is not generic, but that answer is typically nonminimal. (For a minimal solution see Section 4). The answer is found by deforming the non-generic ideal $M$ to a "nearby" generic ideal $M_{\epsilon}$, where $\epsilon$ represents a small positive real. For instance,

$$
M=\left\langle x^{2}, x y, x z, y^{2}, y z, z^{2}\right\rangle \text { deforms to } M_{\epsilon}=\left\langle x^{2}, x^{1+\epsilon} y, x z^{1-\epsilon}, y^{2}, y^{1+\epsilon} z, z^{2}\right\rangle \text {. }
$$

We can apply Theorem 1 to the generic ideal $M_{\epsilon}$ and then set $\epsilon=0$ to get the Hilbert series, a choice of minimal S-pairs, and the irreducible decomposition of $M$. For instance, the irreducible decomposition of the deformation equals

$$
M_{\epsilon}=\left\langle x^{2}, y, z^{1-\epsilon}\right\rangle \cap\left\langle x^{1+\epsilon}, y^{2}, z^{1-\epsilon}\right\rangle \cap\left\langle x, y^{2}, z\right\rangle \cap\left\langle x, y^{1+\epsilon}, z^{2}\right\rangle
$$

We invite the reader to draw the graph $G_{M_{\epsilon}}$ and see what happens for $\epsilon \rightarrow 0$.

The preceding discussion allows Theorem 2 to provide the following complexity result for all monomial ideals in $\mathbb{K}[x, y, z]$. Recall that a planar graph $G_{M}$ on $r$ vertices has at most $3 r-6$ edges and at most $2 r-5$ bounded regions.

\section{Corollary 2.}

1. The numerator of the Hilbert series of an ideal generated by $r$ monomials in three variables $x, y, z$ has at most $6 r-10$ summands.

2. When applying Buchberger's Criterion to $r$ polynomials in three variables, it suffices to form at most $3 r-6$ S-pairs instead of all $\left(\begin{array}{l}r \\ 2\end{array}\right)$ possible $S$-pairs. 


\section{Three Variables: the Non-generic Case}

In this section we outline a proof for the following solution to (i), (ii), and (iii):

Theorem 3. Every monomial ideal $M$ in $\mathbb{K}[x, y, z]$ has a minimal resolution by the bounded regions of a planar graph. That resolution gives irredundant formulas for the numerator of the Hilbert series and the irreducible decomposition of $M$.

For generic monomial ideals $M$ this was established in Theorem 1. Before discussing the proof for arbitrary $M$, we must first explain the meaning of "minimal resolution by a planar graph" $G$. Suppose the vertices of $G$ are labeled by the minimal generators $m_{1}, \ldots m_{r}$ of $M$. Label each edge $\{i<j\}$ of $G$ by $m_{i j}=\operatorname{lcm}\left(m_{i}, m_{j}\right)$. Now $G$, determines a set $T$ of bounded regions, with each region $R \in T$ having a certain set of vertices $\left\{i_{1}<\cdots<i_{t}\right\}$ on its boundary. We define the label $m_{R}=\operatorname{lcm}\left(m_{i_{1}}, \ldots, m_{i_{t}}\right)$. Finally, for each region $R$ and each edge $\{i, j\}$ define the $\operatorname{sign} \varepsilon(R, i j)$ to be 0 unless $\{i, j\}$ is on the boundary of $R$, then 1 if $R$ is on the left as one goes from $i$ to $j$ and -1 if $R$ is on the right.

In analogy with Eq. (8), consider the following maps of free modules:

$$
\begin{aligned}
& \partial_{1}: \mathbb{K}[x, y, z]^{G} \rightarrow \mathbb{K}[x, y, z]^{r}, \quad e_{i j} \mapsto \frac{m_{i j}}{m_{j}} \cdot e_{j}-\frac{m_{i j}}{m_{i}} \cdot e_{i} \\
& \partial_{2}: \mathbb{K}[x, y, z]^{T} \rightarrow \mathbb{K}[x, y, z]^{G}, \quad e_{R} \mapsto \sum_{\{i, j\} \in G} \varepsilon(R, i j) \cdot \frac{m_{R}}{m_{i j}} \cdot e_{i j}
\end{aligned}
$$

which define a complex $\mathbb{F}_{G}$ of free $\mathbb{K}[x, y, z]$-modules in analogy with Eq. (10). Theorem 3 says that it is always possible to choose a labeled planar graph $G$ so that $\mathbb{F}_{G}$ is exact and minimal, i.e., $m_{i j} \neq m_{R}$ for any edge $i j$ of a region $R$. The graph $G$ will be far from unique for monomial ideals $M$ which are not generic.

Example. Consider the $m^{\text {th }}$ power of the maximal ideal $\langle x, y, z\rangle$, that is,

$$
M=\langle x, y, z\rangle^{m}=\left\langle x^{i} y^{j} z^{k}: i, j, k \in \mathbb{N}, i+j+k=m\right\rangle .
$$

The staircase of $M$ is depicted in Figure 3(a) for $m=5$. The graph in Figure 3(b) represents a free resolution of $M$ for $m=5$. This graph is essentially the edge graph of the hull polytope $P_{M}$ of $M$, as defined in Section 3 . In fact, the hull polytope makes sense for any monomial ideal (in any number of variables) and gives a nice but nonminimal solution to our problems (i), (ii), and (iii):

The resolution defined by the graph in Figure $3(\mathrm{~b})$ is nonminimal because every downward-pointing triangle has the same label as all three of its edges. We get a graph $G$ satisfying Theorem 3 by deleting precisely one edge from each downward-pointing triangle. When an edge is deleted, the two adjacent regions join together, as the one with the larger label "swallows" the one with the smaller label. Notice how many distinct graphs $G$ result that minimally resolve $M$ !

Since the monomial ideal $M$ is invariant under the symmetric group on $x, y, z$, we may ask whether there exists a minimal planar graph resolution which is 


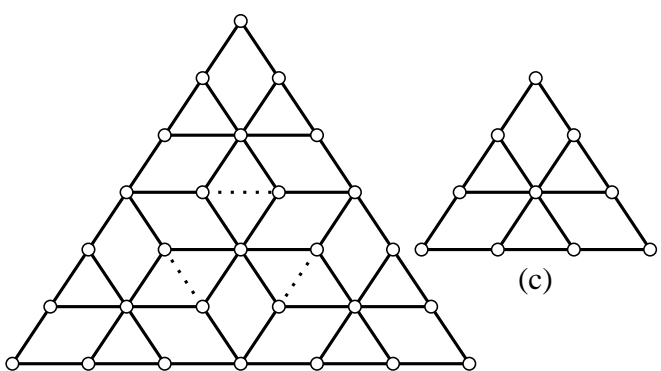

(d)

(e)

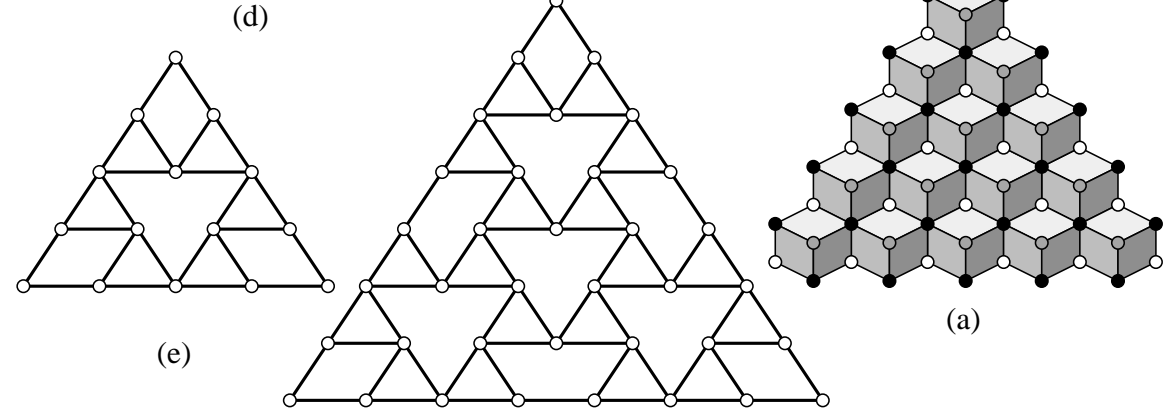

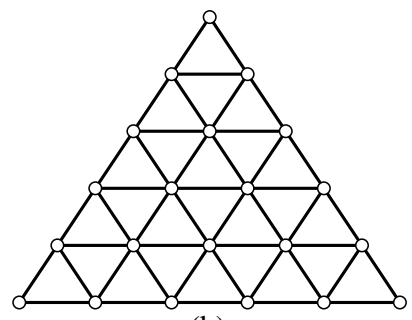

(b)

(f)

Fig. 3. Symmetric resolutions of various powers of the maximal ideal $\langle x, y, z\rangle$

invariant. The answer is "yes" if and only if $m$ is congruent to 0 or 1 modulo 3 . Examples of these symmetric choices are shown for various $m$ in Figure 3: (c) is $m=3$; (d) is, without the dotted edges, $m=6$; (e) is $m=4$; and (f) is $m=7$.

The general construction for the case $m \equiv 0(\bmod 3)$ is gotten by putting together $m^{2} / 9$ of the graphs in Figure 3(c) in the pattern of Figure 3(b), erasing the dotted edges as in Figure 3(d) when everything is put together. In the case $m \equiv 1(\bmod 3)$, one radiates upside-down side-length 2 triangles outward from the center, cutting them off at the boundary as in Figures 3(e,f). Of course, the upside-down side-length 2 "triangles" are really hexagonal regions in the graph.

Proof sketch of Theorem 3. As in the generic case, we may assume that $M$ is artinian. We can always find some planar graph $G^{\prime}$ such that $\mathbb{F}_{G^{\prime}}$ is a (possibly nonminimal) free resolution of $M$, e.g. by using the hull polytope or by deforming to nearby generic monomial ideal. Since the labels on the vertices of $G^{\prime}$ are minimal generators, the matrix $\partial_{1}$ never has \pm 1 entries. The matrix $\partial_{2}$ has a \pm 1 entry if and only if some region has the same label as one of its boundary edges. One shows that the graph obtained from $G^{\prime}$ by deleting this edge and joining the two adjacent regions still defines a free resolution of $M$. A graph $G$ satisfying Theorem 3 is obtained when there are no nonminimal edges left to delete.

Once we know that $\mathbb{F}_{G}$ is a minimal free resolution then the irredundant irreducible decomposition of $M$ can be read off the labels on the regions of $G$, in analogy with Eq. (11). The Hilbert series of $M$ can be written down in analogy 
with Eq. (9). None of the monomials in the resulting formula for the numerator cancel. This very last fact relies on $n \leq 3$ and may be false for $n \geq 4$ variables.

We claim that the following converse to Theorem 3 holds.

Theorem 4. For every 3-connected planar graph $G$ there exists a monomial ideal $M$ in 3 variables which is minimally resolved by the bounded regions of $G$.

This result is a variant of Steinitz' Theorem [19, Theorem 4.1] which states that 3-connected planar graphs are the edge graphs of 3-dimensional convex polytopes. If $G$ is a planar triangulation then $M$ can be chosen generic, and, in this case, Theorem 4 follows from Schnyder's Theorem on order dimension [17, Theorem 6.2.1, pp. 128], as explained in [3, §6]. The general non-triangulated case is more difficult. It does not immediately follow from its order-theoretic analogue due to Brightwell and Trotter [17, Theorem 6.3.1]. The complete proof of Theorem 4 is "under construction" and will be published elsewhere.

In Figure 4 is a non-trivial example illustrating the encoding of a planar graph $G$ by a monomial ideal $M$. Note that the order 8 (dihedral group) symmetry of the graph cannot be reproduced in the monomial ideal. The square is realized by having an irreducible component which is determined by four surrounding generators, two of which have one coordinate in common. Similarly, the hexagons have six generators spread around the corresponding irreducible component, with each such generator sharing one coordinate with the irreducible component. Only the artinian components - those with generators on all three sides - define regions in $G$. After $M$ has been chosen to realize $G$, it may be that $G$ can be altered while still remaining a minimal resolution for $M$. For instance, edge $a$ can have its exterior vertex changed from 1 to 2 , or even to 3 , making the left hexagon into a heptagon or octagon. Independently, edge $b$ can have its vertex 4 moved to 5 , making the right hexagon into a pentagon. What must remain constant are the numbers of vertices, edges, and regions.

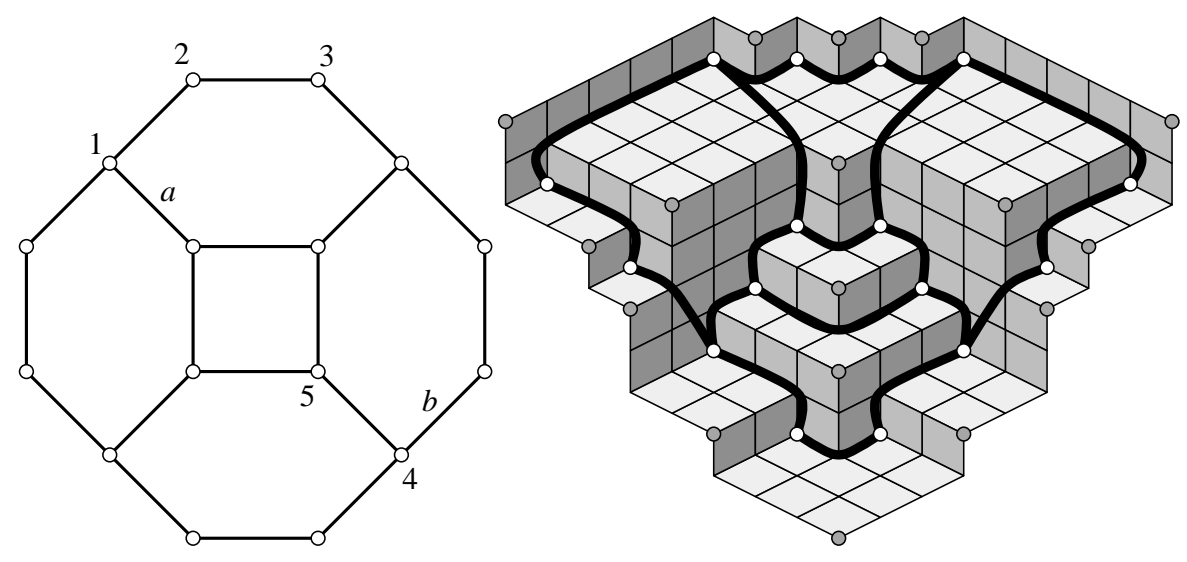

Fig. 4. A monomial ideal constructed to have the given graph as minimal resolution 


\section{$5 \quad$ Four and More Variables}

The ideas presented in Sections 3 and 4 make perfect sense also for $n \geq 4$ variables. There is the hull polytope $P_{M}$ in $\mathbb{R}^{n}$, and there are deformations to generic monomial ideals $M \rightarrow M_{\epsilon}$. Resolutions by planar graphs can be generalized to resolutions by cell complexes, as defined by Bayer and Sturmfels [5]. The familiar duality of planar graphs now becomes Alexander duality for monomial ideals as defined by Miller [15], and yields an efficient algorithm for problem (iii). However, it is more difficult to construct cellular resolutions which are minimal. There seems to be no $n$-dimensional analogue to Theorem 3 . One obstruction is that the minimal resolution may depend on the characteristic of the field $\mathbb{K}$. Any general formula for minimal resolutions is expected to involve homological algebra over the lcm-lattice of Gasharov, Peeva and Welker [12]. See the recent work of Yuzvinsky [18] for a possible approach.

Cellular resolutions provide efficient formulas for Hilbert series and related questions even if they are nonminimal. The following complexity result holds.

Theorem 5. Fix $n \geq 2$. The numerator of the Hilbert series of an ideal $M$ generated by $r$ monomials in $n$ variables can have order $r^{\left\lfloor\frac{n}{2}\right\rfloor}$ many terms for $r \gg 0$. The same upper and lower bound holds for the number of irreducible components of $M$ and the ranks of the modules in a minimal resolution of $M$.

This upper bound was derived by Bayer, Peeva and Sturmfels [3, Theorem 6.3] from the Upper Bound Theorem for Convex Polytopes [19, Theorem 8.23]. The Upper Bound Theorem states, roughly speaking, that the maximum number of faces of an $n$-dimensional polytope with $r$ vertices has order $r^{\left\lfloor\frac{n}{2}\right\rfloor}$. The matching lower bound for Theorem 5 was established by Agnarsson [1].

There seems to be no analogue to Theorem 4 is higher dimensions. For instance, there is a triangulation of a tetrahedron with three interior vertices [3, $\S 6]$ which does not support a minimal resolution of a monomial ideal $M$ in $R=\mathbb{K}[a, b, c, d]$. A detailed study of the graphs $G_{M}$ for $n=4$ appears in recent work of Agnarsson, Felsner and Trotter [2]. The following example is instructive:

$$
\begin{aligned}
M= & \left\langle a^{9} b^{9} c^{9}, d^{9}, a^{6} b^{7} c^{4} d, a^{2} b^{3} c^{8} d^{5}, a^{5} b^{8} c^{3} d^{2}\right. \\
& \left.a b^{4} c^{7} d^{6}, a^{8} b^{5} c^{2} d^{3}, a^{4} b c^{6} d^{7}, a^{7} b^{6} c d^{4}, a^{3} b^{2} c^{5} d^{8}\right\rangle
\end{aligned}
$$

Its S-pair graph $G_{M}$ is the complete graph on 12 vertices. The minimal resolution of $M$ is cellular and looks like $0 \rightarrow R^{53} \rightarrow R^{108} \rightarrow R^{66} \rightarrow R^{12} \rightarrow M \rightarrow 0$. It is a triangulation of a tetrahedron with 8 interior vertices, which is neighborly in the sense that any two vertices form an edge. Polytope combinatorics [19, §8] tells us that such a neighborly triangulation has 53 tetrahedra and 108 triangles.

Let $\phi(n)$ denote the maximum of generators of a monomial ideal $M$ in $n$ variables whose S-pair graph $G_{M}$ equals the complete graph. It is a non-trivial fact that $\phi(n)$ is actually finite. However, it grows doubly-exponentially in $n$; see [17, Theorem 7.2.13]. Note that Corollary 1 implies $\phi(2)=2$, Corollary 2 implies $\phi(3)=4$, and $\phi(4)=12$ is attained by the neighborly monomial ideal $M$ above. The specific values in Table 1 are derived from the following theorem. 
Theorem 6. (Hosten and Morris [13]) The number $\phi(n)$ equals the number of distinct simplicial complexes $T$ on the set $\{1,2, \ldots, n-1\}$ with the property that no two faces of $T$ have their union equal to $\{1,2, \ldots, n-1\}$.

Table 1. The maximum number $\phi(n)$ of generators of a neighborly monomial ideal

\begin{tabular}{r|ccccccc} 
variables $=\mathrm{n}$ & 2 & 3 & 4 & 5 & 6 & 7 & 8 \\
\hline generators $=\phi(n)$ & 2 & 4 & 12 & 81 & 2,646 & $1,422,564$ & $229,809,982,112$
\end{tabular}

\section{References}

1. G. Agnarsson. On the number of outside corners of monomial ideals, Journal of Pure and Applied Algebra, 117 (1997) 3-22.

2. G. Agnarsson, S. Felsner and W. Trotter. The maximum number of edges in a graph of bounded dimension, with applications to ring theory, Discrete Mathematics (201) (1999) 5-19

3. D. Bayer, I. Peeva and B. Sturmfels. Monomial resolutions, Mathematical Research Letters 5 (1998) 31-46.

4. D. Bayer and M. Stillman. Computation of Hilbert functions, Journal of Symbolic Computation 14 (1992), 31-50.

5. D. Bayer and B. Sturmfels. Cellular resolutions of monomial modules. Journal für die reine und angewandte Mathematik, 502 123-140, 1998.

6. A. Bigatti, M. Caboara and L. Robbiano. On the computation of Hilbert-Poincaré series. Appl. Algebra Engrg. Comm. Comput. 2 (1991), no. 1, 21-33.

7. B. Buchberger, A criterion for detecting unnecessary reductions in the construction of Gröbner bases, Proc. EUROSAM 79, Marseille, W. Ng (ed.), Lecture Notes in Computer Science, Vol. 72, Springer 1979, pp. 3-21.

8. M. Caboara, P. Conti and C. Traverso, Yet another ideal decomposition algorithm. Applied algebra, algebraic algorithms and error-correcting codes (Toulouse, 1997), Lecture Notes in Computer Science, Vol. 1255, Springer, 1997, pp. 39-54,

9. A. Capani, G. de Dominicis, G. Niesi and L. Robbiano. Computing minimal finite free resolutions, Journal of Pure and Applied Algebra 117/118 (1997), 105-117.

10. D. Cox, J. Little and D. O'Shea, Ideals, Varieties, and Algorithms, 2nd edition, Springer-Verlag, New York, 1997

11. D. Eisenbud. Introduction to Commutative Algebra with a View towards Algebraic Geometry, Springer Verlag, New York, 1995.

12. V. Gasharov, I. Peeva and V. Welker, The lcm-lattice, preprint, 1998.

13. S. Hosten and W. Morris Jr. The order dimension of the complete graph, Discrete Mathematics (201) (1999), 133-139.

14. R. La Scala and M. Stillman. Strategies for computing minimal free resolutions, Journal of Symbolic Computation 26 (1998), no. 4, 409-431.

15. E. Miller. Alexander duality for monomial ideals and their resolutions, preprint, 1998.

16. H.M. Möller and F. Mora. New constructivge methods in classical ideal theory, Journal of Algebra 100 (1986) 138-178.

17. W. Trotter. Combinatorics and Partially Ordered Sets. Dimension Theory, The Johns Hopkins University Press, Baltimore, 1992.

18. S. Yuzvinsky, Taylor and minimal resolutions of homogeneous polynomial ideals, preprint, 1999.

19. G. Ziegler. Lectures on Polytopes, Springer Verlag, New York, 1994. 